PAPER • OPEN ACCESS

\section{Post-collision interaction effect in THz-assisted Auger decay of noble gas atoms}

To cite this article: I J Bermúdez Macias et al 2021 J. Phys. B: At. Mol. Opt. Phys. 54085601

View the article online for updates and enhancements.
You may also like

- The LVV Auger line shape of sulfur on
copper studied by Auger photoelectron
coincidence spectroscopy
G Di Filippo, M I Trioni, G Fratesi et al.
- Discovery of ASKAP J173608.2-321635
as a Highly Polarized Transient Point
Source with the Australian SKA Pathfinder
Ziteng Wang, David L. Kaplan, Tara
Murphy et al.
- Exact solution of the two-axis
countertwisting hamiltonian for the half-
integer J case
Feng Pan, Yao-Zhong Zhang and Jerry P
Draayer




\title{
Post-collision interaction effect in THz-assisted Auger decay of noble gas atoms
}

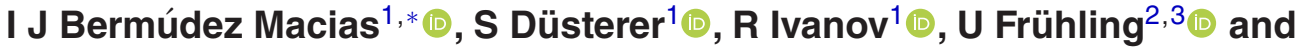 \\ N M Kabachnik ${ }^{1,4,5}$
}

${ }^{1}$ Deutsches Elektronen-Synchrotron (DESY), Notkestrasse 85, D-22603 Hamburg, Germany

2 Institute for Experimental Physics, University Hamburg, Hamburg, Germany

3 The Hamburg Centre for Ultrafast Imaging, Luruper Chaussee 149, Hamburg, Germany

${ }^{4}$ Donostia International Physics Center (DIPC), E-20018, San Sebastian/Donostia, Spain

5 Skobeltsyn Institute of Nuclear Physics, Lomonosov Moscow State University, Moscow 119991, Russia

E-mail: ivette.bermudez@desy.de

Received 12 November 2020, revised 28 February 2021

Accepted for publication 23 March 2021

Published 3 May 2021

\begin{abstract}
Auger electron spectra were simulated in the presence of a terahertz streaking field to study post-collision interaction (PCI) effects in the time-evolution of photoinduced Auger decays. The PCI is characterized by the ratio of the spectral line width for Auger electron emission in opposite directions with respect to the THz-field. These calculations have been performed using the analytical semiclassical model developed by Bauch and Bonitz (2012 Physical Review A 85 053416). The results are shown for $\mathrm{Ne}(\mathrm{KLL}), \operatorname{Ar}(\mathrm{LMM}), \mathrm{Kr}(\mathrm{MNN})$ and $\mathrm{Xe}(\mathrm{NOO})$ Auger transitions whereby different possible experimental conditions were evaluated.
\end{abstract}

Keywords: post-collision interaction, Auger decay, terahertz streaking

(Some figures may appear in colour only in the online journal)

\section{Introduction}

The term 'post-collision interaction (PCI)' encompasses a broad range of phenomena in atomic collisions and photoionization, associated with the influence of the Coulomb interaction of slowly receding charged particles on autoionization (Auger) electron spectra [1]. In particular, PCI effects were observed in near-threshold inner shell photoionization of atoms with the following Auger decay (see [2, 3] and references therein). Here, the interaction with the slow photoelectron shifts the energy of the Auger electron and considerably distorts the Auger line-shape in the spectrum. In addition, PCI

* Author to whom any correspondence should be addressed.

cC) (i) Original content from this work may be used under the terms of the Creative Commons Attribution 4.0 licence. Any further distribution of this work must maintain attribution to the author(s) and the title of the work, journal citation and DOI. is responsible for the recapture of emitted photoelectrons to Rydberg states of the ions [4]. These phenomena were thoroughly studied experimentally in nineties and zeroes at synchrotron radiation facilities (see, for example, [5-9] as well as references in $[2,3])$. A good understanding of the PCI effects was achieved first on a semiclassical basis [10-13] and later in a stationary quantum mechanical picture of the process within the unified theory of the Auger process $[14,15]$ and the random phase approximation with exchange approach $[16,17]$. The non-stationary description of the PCI-induced recapture process was developed in $[9,18]$.

The above mentioned experiments were performed by standard time-integrated spectroscopic methods using highresolution electron spectrometers. In the past decades, tremendous progress in the availability of femtosecond and subfemtosecond photon pulses has enabled the study of atomic processes in the time-domain, in particular Auger decays [19-25]. A first terahertz (THz)-assisted time-resolved study 
of the Auger process in atoms has revealed that PCI leads to a time-dependent (chirped) Auger electron energy [26].

In the experiment [26], $\mathrm{Kr} \mathrm{MNN}$ and Xe NOO Auger spectra, excited by ultrashort (femtosecond) extreme ultraviolet (XUV) pulses from the free-electron laser in Hamburg (FLASH) [27] and from a high harmonic laser source have been studied. The electrons were ionized in the presence of a co-propagating, linearly polarized $\mathrm{THz}$ field which introduced a time dependent modulation (streaking) of the electron energies. By changing the time delay between XUV and $\mathrm{THz}$ pulses the time development of the Auger decay has been investigated. A significant difference of the widths of kinetic energy spectra for opposite observation directions and $\mathrm{THz}$ field gradients has been observed. This indicates an energetic chirp, i.e. a pronounced time-dependent variation of the Auger electrons kinetic energy. Theoretical interpretation of the results has been given using the semiclassical approach developed by Bauch and Bonitz [28]. It was shown that the chirp may be explained by the PCI of the photo- and Auger electrons in the final state. Later this finding has been used to study the temporal evolution of interatomic-Coulombic decay by evaluating the PCI energy shift of electrons and thereby deducing the ionization times [29]. Moreover, it was demonstrated that the measured PCI effect is very sensitive to the details of the photoinduced Auger emission. Furthermore, it was suggested to use the observed effect in metrology of ultra-fast pulses. These observations stimulate an interest in continuing and broadening the studies of the time-resolved PCI effects. Thus, the present paper shall serve as a basis for experimental studies on FELs or HHG sources in order to determine the optimum experimental conditions to observe a strong PCI effect. In particular, the study is motivated to guide future experiments at the THz streaking setup at FLASH $[30,31]$.

In the present paper we report the results of the theoretical study of the THz-assisted photoinduced Auger processes in noble gas atoms. The main goal is to investigate the PCI effects in the process and their dependence on the experimental parameters. We used the analytical model of the process developed by Bauch and Bonitz [28]. A concise description of the model is presented in section 2. The calculations have been done for KLL transitions in Ne, LMM in Ar, MNN in $\mathrm{Kr}$ and $\mathrm{NOO}$ in $\mathrm{Xe}$. The results are shown and discussed in section 3. Finally, section 4 contains the conclusions and outlook.

\section{Semiclassical description of $\mathrm{PCI}$}

An accurate theoretical description of the $\mathrm{THz}$ assisted photoinduced Auger process might be given on the basis of a numerical solution of the time-dependent Schrödinger equation [32] which involves both the exciting XUV pulse and the streaking $\mathrm{THz}$ pulse. This approach would automatically include the description of PCI as it was demonstrated in [18] for the case without a THz pulse. However, the long streaking $\mathrm{THz}$ pulse in comparison with the fast oscillations of the electronic wave functions makes a direct application of this method not feasible due to prohibitively long computation time. Bauch and Bonitz [28] suggested a simple analytical model which incorporates both the PCI effect and the streaking in the $\mathrm{THz}$ field. The model is based on the semiclassical description of the PCI effect $[10,12,13]$. Namely, it is associated with the abrupt change of the screening which is felt by the slowly receding photoelectron at the moment when the fast Auger electron overtakes it. The movement of both electrons is considered classically, and an essential parameter of the model is the distance from the ion nucleus at which the Auger electron overtakes the photoelectron. The model was tested in [28] by comparing it with the solution of the time-dependent one-dimensional Schrödinger equation and Monte Carlo molecular dynamics simulations. Furthermore, it has accurately described the experimental data [26].

In the following, we use the analytical semiclassical model [28] to predict the PCI effects in THz assisted Auger decay in noble gas atoms. In a short description of the model we follow the paper [28].

Consider a photoelectron emitted at a time $t_{\mathrm{ph}}$ with a momentum $p_{\mathrm{ph}}$ and an Auger electron released after the photoelectron at a time $\tau_{\mathrm{A}}$ with a momentum $p_{\mathrm{A}}$ in the presence of a linearly polarized streaking $\mathrm{THz}$ field with vector potential $A(t)$. It is supposed that $p_{\mathrm{A}}>p_{\mathrm{ph}}$. The Auger decay line-width (without $\mathrm{THz}$ field) is denoted $\Gamma_{\mathrm{A}}$ so that the core-hole life-time is $\Gamma_{\mathrm{A}}^{-1}$ (here and below atomic units are used unless otherwise indicated). For calculating the Auger-electron spectral line-shape, the $\mathrm{THz}$ vector potential is expanded around the moment of the photoemission in Taylor series:

$$
A\left(\tau_{\mathrm{A}}\right)=A_{P}+\dot{A}_{P} \tau_{\mathrm{A}}+\mathcal{O}\left(\tau_{\mathrm{A}}^{2}\right),
$$

where $A_{P}=A\left(t_{\mathrm{ph}}\right)$ while $\dot{A}_{P}=\frac{\mathrm{d} A}{\mathrm{~d} t}\left(t_{\mathrm{ph}}\right)$ denotes the first time derivative of the vector potential. We consider the Auger electron spectra in two neighbouring zero-crossings of the vector potential where $A_{P}=0$. Ignoring the higher order terms of the expansion (1), we obtain that the energy shift for Auger electrons in the THz field $p_{\mathrm{A}} A\left(\tau_{\mathrm{A}}\right)=p_{\mathrm{A}} \dot{A}_{P} \tau_{\mathrm{A}}$ depends on the sign of the derivative $\dot{A}_{P}$. Moreover, it is clear that the streaking effect for different signs of $\dot{A}_{P}$ can be equivalently studied by changing the sign of $p_{\mathrm{A}}$, i.e. by detecting the emitted Auger electrons in opposite directions.

The model Auger spectrum generated by the XUV pulse in the $\mathrm{THz}$ field is presented as the numerical convolution of the PCI-distorted spectral line shapes $f \pm(\epsilon)$ with the streaked energy spectrum $f_{X}(\hat{\epsilon})$ due to the finite XUV pulse duration:

$$
f_{X \pm}(\epsilon)=\int_{-\infty}^{+\infty} \mathrm{d} \hat{\epsilon} f_{X}(\hat{\epsilon}) f \pm(\epsilon-\hat{\epsilon}) .
$$

Here

$$
f_{X}(\hat{\epsilon})=\frac{1}{\sqrt{\pi} \Gamma_{X}} \exp \left(-\frac{\hat{\epsilon}^{2}}{\Gamma_{X}^{2}}\right)
$$

with

$$
\Gamma_{X}=p_{\mathrm{A}} \dot{A}_{P} \tau_{X}
$$

where $\tau_{X}$ is the XUV pulse duration full-width at halfmaximum (FWHM). 
As demonstrated in [28], for a positive derivative of the streaking electric field $\dot{A}_{P}>0$, the spectral line shape $f_{+}(\epsilon)$ may be presented as:

$$
f_{+}(\epsilon)=\Gamma_{4} \frac{k_{+}-\epsilon_{-}}{k_{+}} e^{-\Gamma_{4}\left(k_{+}-\epsilon_{+}\right)},
$$

where $\Gamma_{4}=|\alpha| \Gamma_{A}$ with $\alpha=\left(2 p_{\mathrm{A}} \dot{A}_{P}\right)^{-1}$.

On the other hand, for negative values of $\dot{A}_{P}<0$, the Auger spectral line shape is:

$$
f_{-}(\epsilon)=2 \Gamma_{4} e^{-\Gamma_{4} \epsilon_{+}}\left(\frac{\epsilon_{-}}{k_{-}} \cosh \Gamma_{4} k_{-}-\sinh \Gamma_{4} k_{-}\right) .
$$

In these equations $k_{ \pm}=\sqrt{\epsilon_{-}^{2} \pm|\beta|}, \epsilon_{ \pm}=\epsilon \pm \frac{\beta}{4} \delta r^{*}$ with $\beta=4 p_{\mathrm{A}} \dot{A}_{P} / p_{r}$.

The parameters $p_{r}$ and $\delta r *$ determine the classical distance from the ion at which the Auger electron overtakes the slower photoelectron:

$$
r^{*}=\tau_{\mathrm{A}} p_{r}+\delta r^{*}
$$

Here $p_{r}=p_{\mathrm{A}} p_{\mathrm{ph}} /\left[p_{\mathrm{A}}-p_{\mathrm{ph}}+A\left(t_{\mathrm{ph}}\right)-A\left(\tau_{\mathrm{A}}\right)\right]$, and $\delta r^{*}$ is a small correction to the initial distance of the two electrons from the ion. For the derivation of these expressions and their detailed discussion the reader is referred to the paper [28].

As it is shown in [28], due to the influence of PCI the spectral width of the Auger line at $\dot{A}>0$ (denoted $\omega_{+}$) is larger than at $\dot{A}<0$ (denoted $\omega_{-}$).

Qualitatively, the difference in spectral width at the two slopes of the vector potential may be explained as follows. The energy shift of the Auger electron in the THz field taking into account the PCI effect is $\Delta E_{\mathrm{A}}=-p_{\mathrm{A}} A(t)+A^{2}(t)+E_{\mathrm{PCI}}(t)$, where $t$ is the moment of the Auger electron emission. The term $E_{\mathrm{PCI}}(t)$ accounts for the change of Auger electron energy due to the PCI effect. In the semiclassical model, this term is equal to the energy gain of the Auger electron when it overtakes the slow photoelectron at the distance $r^{*}(t)$. It is due to the change of screening at this moment and is equal to $E_{\mathrm{PCI}}(t)=$ $1 / r^{*}(t)$. When time $t$ increases, the Auger electron overtakes the photoelectron at a larger distance and therefore gains less energy. Thus the energy of the Auger electron depends on time indicating a 'chirp'. The quadratic term $A^{2}(t)$ is small and may be ignored. The width induced by the THz field and the PCI may be estimated as $\delta\left(\Delta E_{\mathrm{A}}\right)=\left(-p_{\mathrm{A}} \mathrm{d} A / \mathrm{d} t+\mathrm{d} E_{\mathrm{PCI}} / \mathrm{d} t\right) \Gamma^{-1}$. The second term does not depend on the sign of $\mathrm{d} A / \mathrm{d} t$ and is always negative. Therefore, for a positive derivative both terms have the same sign and the width is larger than for a negative one, where the two terms have different signs and partly cancel each other. This is the consequence of the chirp and is similar to the effect of chirp on the spectral width of photoelectrons [33]. On the other hand, these widths are equal if the PCI effect is negligible. Therefore, it is convenient to characterize the PCI effect by the ratio of the widths $\omega_{+} / \omega_{-}$.

\section{Results and discussion}

To illustrate the PCI effect for experimentally reachable parameters at FELs or HHG sources in the time evolution of the Auger decay, the photon energies were varied such that the ratio of the photoelectron to the Auger electron energy ranged between zero (the photon energy is equal to the corresponding threshold energy) and one (the photoelectrons have the same energy as the Auger electrons). Therefore, we have chosen the following representative examples of the Auger transitions:

1. $\mathrm{KL}_{2,3} \mathrm{~L}_{2,3}\left({ }^{1} D_{2}\right)$ transition in Ne. This is the strongest line in Ne Auger spectrum at the Auger electron energy of $804.5 \mathrm{eV}$ [34]. The binding energy of the $1 \mathrm{~s}$ electron is $870.2 \mathrm{eV}$ and the decay width is $\Gamma_{\mathrm{A}}=263.2 \mathrm{meV}$ [35] corresponding to a lifetime of $\Gamma_{\mathrm{A}}^{-1}=2.5 \mathrm{fs}$. The photon energy needed for the experiment is in the interval from 870.2 to $1674.7 \mathrm{eV}$.

2. $\mathrm{L}_{3} \mathrm{M}_{2,3} \mathrm{M}_{2,3}\left({ }^{1} S_{o}\right)$ transition in Ar with an Auger electron energy of $201 \mathrm{eV}$ [36]. The binding energy of the $2 p_{3 / 2}$ electron is $248.4 \mathrm{eV}$ and the decay width is $\Gamma_{\mathrm{A}}=114 \mathrm{meV}$ [36] corresponding to a lifetime of $\Gamma_{\mathrm{A}}^{-1}=5.5 \mathrm{fs}$. The required photon energy should therefore fall between 248.4 and $449.4 \mathrm{eV}$.

3. $\mathrm{N}_{5} \mathrm{O}_{2,3} \mathrm{O}_{2,3}\left({ }^{1} S_{o}\right)$ transition in Xe with an Auger electron energy of $29.8 \mathrm{eV}$ [37]. The binding energy of the $4 d_{5 / 2}$ electron is $67.5 \mathrm{eV}$ and the decay width is $\Gamma_{\mathrm{A}}=100 \mathrm{meV}$ [38] corresponding to a lifetime of $\Gamma_{\mathrm{A}}^{-1}=6.5 \mathrm{fs}$. The required photon energy for the experiment is in the interval from 67.5 to $97.3 \mathrm{eV}$.

4. $\mathrm{M}_{5} \mathrm{~N}_{1} \mathrm{~N}_{2,3}\left({ }^{1} P_{1}\right)$ transition in $\mathrm{Kr}$ that has an Auger electron energy of $38.4 \mathrm{eV}$ [37]. The binding energy of the $3 d_{5 / 2}$ electron is $93.8 \mathrm{eV}$ and the decay width is $\Gamma_{\mathrm{A}}=88 \mathrm{meV}$ [22] corresponding to a lifetime of $\Gamma_{\mathrm{A}}^{-1}=8 \mathrm{fs}$. The required photon energy for the experiment is in the interval from 93.8 to $132.2 \mathrm{eV}$.

$\mathrm{X}$-ray photons with the requisite energy to photoionize $\mathrm{Ne}$ and Ar are available at e.g. European XFEL [39] and LCLS [40], while XUV photons for the photoionization of $\mathrm{Kr}$ and $\mathrm{Xe}$ are available at e.g. FLASH and HHG sources.

For these transitions, we have calculated the ratio of the line width $R_{\omega}=\omega_{+} / \omega_{-}$as a function of the photoelectron energy. The results are presented in figures $1-4$. We note that the value of the parameter $\delta r^{*}$ has no significant effect on the final results. Therefore, in all calculations below, we assumed that $\delta r^{*}=1$ as it was used in [28]. Furthermore, for a fixed streaking field strength, the calculations are independent of the frequency. For each case, we considered $\mathrm{THz}$ field strengths from 30 to $300 \mathrm{kV} \mathrm{cm}^{-1}$ and transform limited XUV (x-ray) photon pulses with Gaussian envelopes and durations from 5 to $100 \mathrm{fs}$ FWHM.

Figure 1 shows the results for the $\mathrm{Ne}(\mathrm{KLL})$ Auger transitions with a core-hole lifetime of $\Gamma_{\mathrm{A}}^{-1}=2.5 \mathrm{fs}$ for a $\mathrm{THz}$ field strength of $120 \mathrm{kV} \mathrm{cm}^{-1}$. When the energy of the photoelectron is smallest (close to zero) compared to that of the Auger electron, the width of the spectral line in the falling slope of the vector potential $(\dot{A}<0)$ is smaller than in the rising slope $(\dot{A}>0)$ and the ratio $R_{\omega}$ is highest. This ratio is decreased by increasing the photoelectron energy and it is one (both widths are the same) when the Auger and photoelectrons have the same energy, meaning that the PCI effect is negligible. Furthermore, the effect can be easier resolved (it is more pronounced) for shorter XUV pulse durations. 


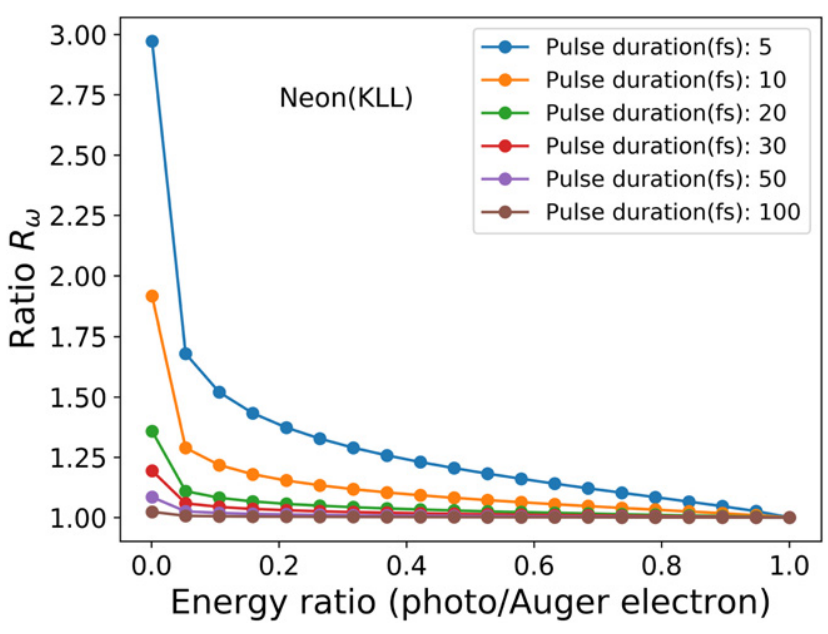

Figure 1. Ratio of the widths $R_{\omega}$ for the neon Auger spectral line $\mathrm{KL}_{2,3} \mathrm{~L}_{2,3}\left({ }^{1} D_{2}\right)$ for different photon pulse durations at a $120 \mathrm{kV}$ $\mathrm{cm}^{-1} \mathrm{THz}^{-1}$ electric field strength. The ratio is maximum when the photoelectron energy is close to zero, and goes to one when both photo and Auger electrons have the same energy.

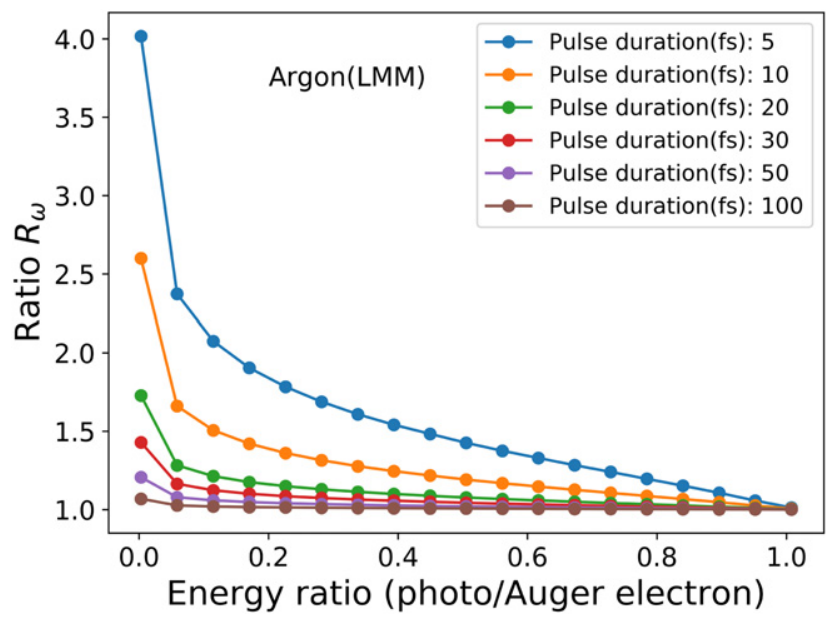

Figure 2. The same as in figure 1 but for the argon Auger spectral line $\mathrm{L}_{3} \mathrm{M}_{2,3} \mathrm{M}_{2,3}\left({ }^{1} S_{0}\right)$.

In figure 2 the results of the $\operatorname{Ar}(\mathrm{LMM})$ Auger transitions with a core-hole lifetime of $\Gamma_{\mathrm{A}}^{-1}=5.5$ fs for a $\mathrm{THz}$ field strength of $120 \mathrm{kV} \mathrm{cm}^{-1}$ are presented. In this case, similarly to the previous one, the ratio of the spectral line widths at the rising and falling slopes of the $\mathrm{THz}$ vector potential is also maximum for relatively slow photoelectrons and goes to one (there is no PCI effect) when both Auger and photoelectrons have the same energy.

The results for the $\mathrm{Xe}(\mathrm{NOO})$ Auger transitions with a corehole lifetime of $\Gamma_{\mathrm{A}}^{-1}=6.5$ fs and for $\mathrm{Kr}(\mathrm{MNN})$ Auger transitions with a core-hole lifetime of $\Gamma_{\mathrm{A}}^{-1}=8$ fs are shown in figures 3 and 4, respectively. In both cases the THz field strength is $120 \mathrm{kV} \mathrm{cm}^{-1}$. Similarly to the previous atoms, the ratio of the widths of the Auger spectral lines in the rising and falling slopes of the $\mathrm{THz}$ vector potential is highest for the slowest photoelectrons and goes to one when both the Auger and photoelectrons have the same energy.

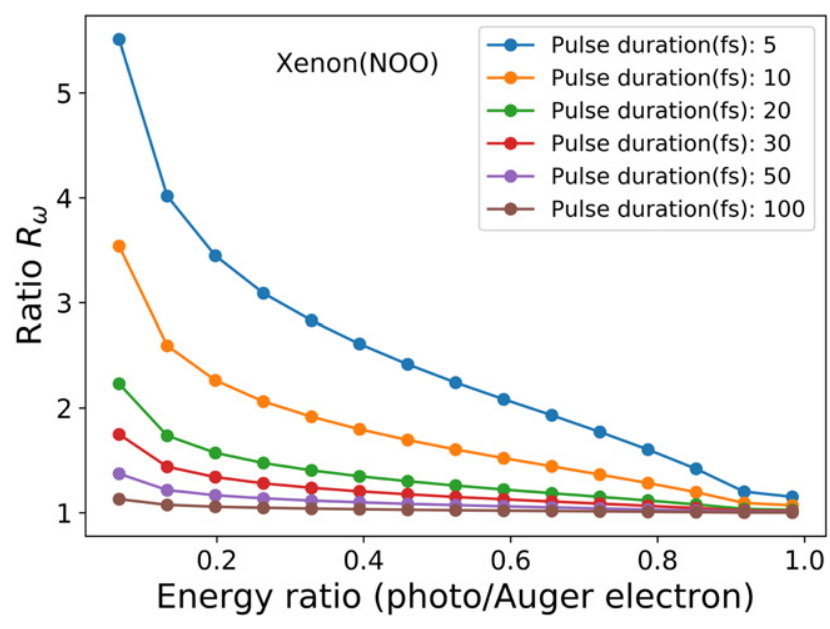

Figure 3. The same as in figure 1 but for the xenon Auger spectral line $\mathrm{N}_{5} \mathrm{O}_{2,3} \mathrm{O}_{2,3}\left({ }^{1} S_{0}\right)$.

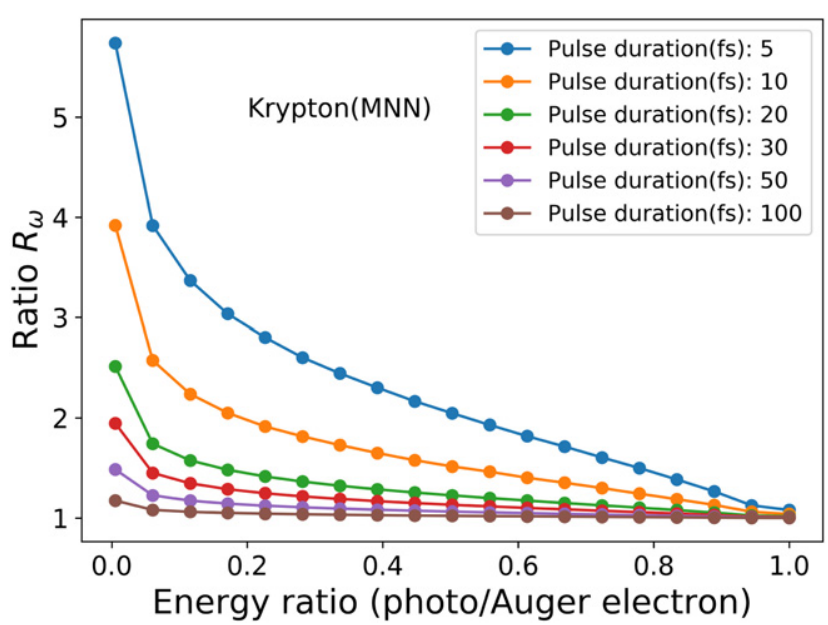

Figure 4. The same as in figure 1 but for the krypton Auger spectral line $\mathrm{M}_{5} \mathrm{~N}_{1} \mathrm{~N}_{2,3}\left({ }^{1} P_{1}\right)$.

As mentioned earlier, calculations were done for different electric field strengths. In figure 5, the results of the simulations for electric field strengths from 60 to $300 \mathrm{kV} \mathrm{cm}{ }^{-1}$ with a photon pulse duration of 20 fs FWHM for $\operatorname{Kr}(\mathrm{MNN})$ Auger transitions are shown. Figure 5(a) shows the ratio $R_{\omega}$ as a function of the energy ratio of the photoelectron to the Auger electron. In figure 5(b) the line widths in $\mathrm{eV}$ at the rising and falling slopes of the vector potential are plotted. As seen from figure 5(b), the Auger line width is larger for stronger streaking THz field. Furthermore, the ratio $R_{\omega}$ in figure 5(a), which characterizes the PCI effect, is largest for the weakest THz field. This may be explained by the fact that the Auger line width in the THz field is determined by both streaking and PCI effects. The streaking effect increases with the $\mathrm{THz}$ field strength, while the PCI effect is independent of the THz field. Therefore, the relative contribution of the PCI effect diminishes with the THz field strength.

Conversely, in order to experimentally measure the PCI effect, one has to account for the experimental resolution of the spectral line widths with an instrument broadening of $\sim 1 \mathrm{eV}$. 


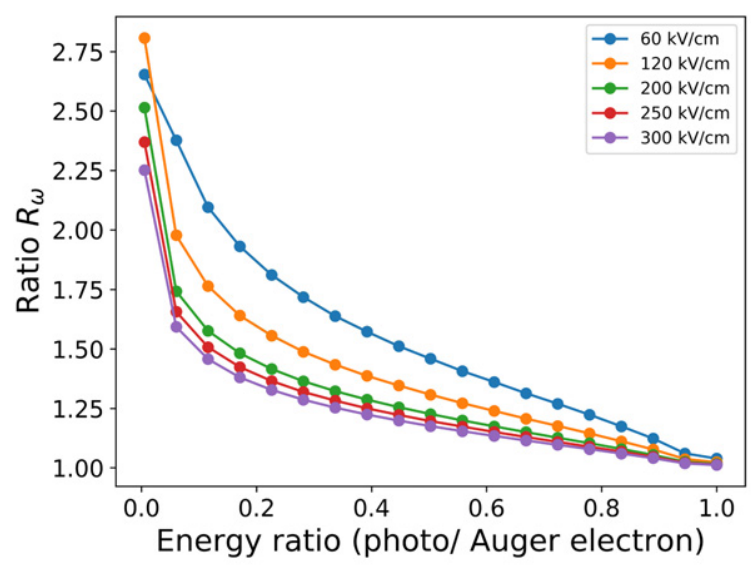

(a)

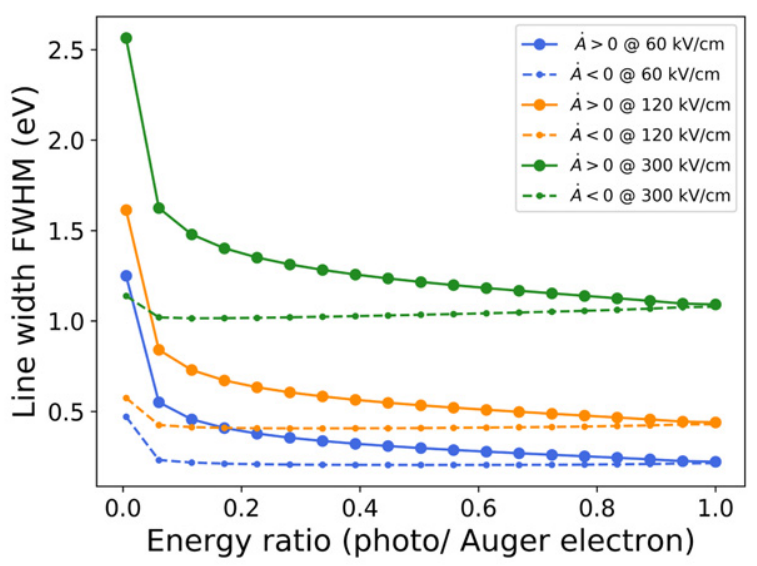

(b)

Figure 5. (a) Ratio $R_{\omega}$ for the krypton Auger spectral line $\mathrm{M}_{5} \mathrm{~N}_{1} \mathrm{~N}_{2,3}\left({ }^{1} P_{1}\right)$ for different $\mathrm{THz}$ streaking field strengths with a photon pulse duration of 20 fs FWHM. (b) Widths in eV of the same krypton spectral line at the rising $\dot{A}>0$ (solid) and falling $\dot{A}<0$ (dotted) slope of the $\mathrm{THz}$ vector potential. Stronger $\mathrm{THz}$ fields streak the pulse more resulting in a broader spectral line.

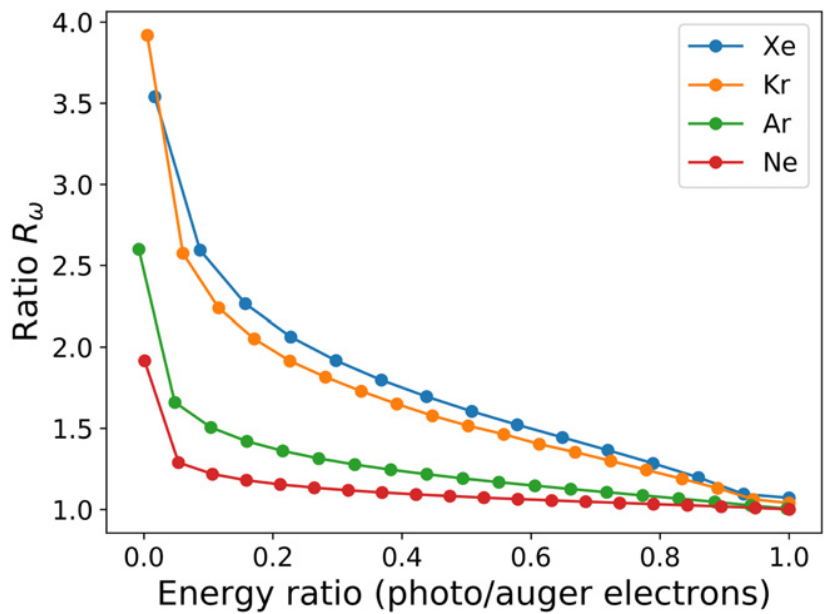

Figure 6. Comparison of the ratio $R_{\omega}$ between xenon, krypton, argon and neon atoms. The calculations were done for a pulse duration of $10 \mathrm{fs}$ and a THz field strength of $120 \mathrm{kV} \mathrm{cm}^{-1}$. The PCI effect is stronger for xenon and krypton atoms compared to argon and neon. The widths' ratio is almost double for the same energy ratio between the photo and Auger electrons. On the other hand, similar behaviour is observed for all of the atoms - the width ratio is maximum for photoelectrons with energy close to zero and it goes to one when both photo and auger electrons have the same energy.

A stronger THz field and thus a broader line width will better resolve the effect. Therefore, the PCI effect $\left(R_{\omega}\right)$ together with the experimental resolution is the parameter that has to be optimized.

A comparison between the four considered transitions is shown in figure 6 for an XUV pulse duration of $10 \mathrm{fs}$ and a $\mathrm{THz}$ field strength of $120 \mathrm{kV} \mathrm{cm}{ }^{-1}$. It is observed that there is a stronger PCI effect for the xenon and krypton atoms as compared to neon and argon. This can be explained by the different core-hole lifetimes. To show this, calculations at a fixed Auger and photoelectron energies but different core-hole lifetimes ranging from zero to $15 \mathrm{fs}$ were done.

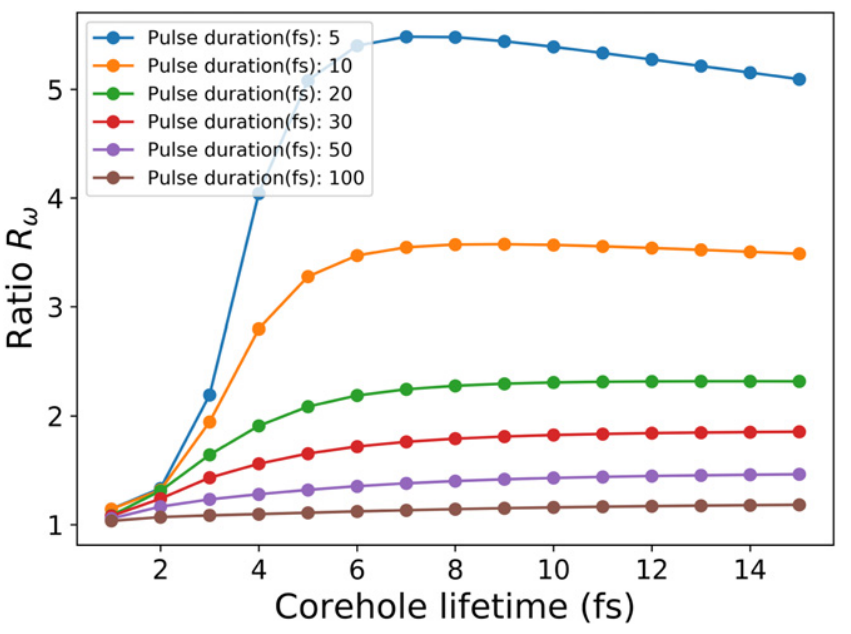

Figure 7. Ratio $R_{\omega}$ of the spectral lines at the rising and falling slopes of the vector potential as a function of its core-hole lifetime. The calculations were done for Auger and photoelectron energies of 30 and $0.5 \mathrm{eV}$ respectively, with a THz field strength of $120 \mathrm{kV} \mathrm{cm}^{-1}$ and various photon pulse durations. The PCI effect is negligible (the ratio is one) for relatively short core-hole lifetimes and increases for values longer than $4 \mathrm{fs}$.

Figure 7 shows the ratio of the spectral line widths at the rising and falling slopes of the $\mathrm{THz}$ vector potential for a field strength of $120 \mathrm{kV} \mathrm{cm}^{-1}$ and different XUV pulse durations at a photo and Auger electron energies of $0.5 \mathrm{eV}$ and $30 \mathrm{eV}$ respectively. For relatively short core-hole lifetimes, the PCI effect is negligible (the ratio is almost one) and it increases significantly for core-hole lifetimes longer than 4 fs and reaches 'saturation' at $7 \mathrm{fs}$. Since the core-hole lifetimes of neon and argon are below $7 \mathrm{fs}$, the effect is not so pronounced in the results shown in figures 1 and 2 as compared to xenon and krypton (figures 3 and 4).

In the semi-classical approach used in our calculations the electron-electron correlations are not included which might influence the accuracy of the results, especially for 
low-energy photoelectrons close to threshold. However, full quantum-mechanical calculations of Auger electron spectrum (without $\mathrm{THz}$ field), which take into account electron correlations $[14,16]$, show excellent agreement with the semiclassical approach also for photoelectrons with very low energy. Moreover, in reference [14] it was shown that the semi-classical approach is valid when the excess energy $E_{\mathrm{ex}}>\left(\Gamma E_{\mathrm{A}}\right)^{1 / 2}$, where $\Gamma$ is the Auger width and $E_{\mathrm{A}}$ the Auger electron energy. For our examples, this condition is satisfied when the ratio of photoelectron and Auger electron energies $E_{\mathrm{e}} / E_{\mathrm{A}}>0.06(\mathrm{Xe}), 0.05(\mathrm{Kr}), 0.02(\mathrm{Ar}, \mathrm{Ne})$. Thus, we suppose that our results are accurate practically for the whole considered energy range with an exception of points close to zero.

\section{Conclusions}

Using the semi-classical analytical model developed in [28], we presented the results of numerical calculations of the PCI effect for different noble gas atoms and various experimental conditions. The results show that there is a stronger effect for transitions with a core-hole lifetime longer than 4 fs, namely xenon and krypton. Furthermore, it is easier to resolve the effect for photon pulse durations shorter than $50 \mathrm{fs}$ FWHM as compared to longer ones. Surprisingly, lower streaking $\mathrm{THz}$ field strengths result in a larger ratio $R_{\omega}$. These results will help future experiments at HHG or FEL sources to tailor experimental conditions, in order to determine a parameter space in which a measurable effect can be expected.

\section{Acknowledgments}

NMK acknowledges the hospitality and financial support from DESY and from the theory group in cooperation with the SQS research group of the European XFEL (Hamburg), as well as the financial support from Donostia International Physics Centre, DIPC (San Sebastian). UF acknowledges financial support from the excellence cluster 'The Hamburg Centre for Ultrafast Imaging - Structure, Dynamics and Control of Matter at the Atomic Scale' (DFG)_EXC 1074 project ID 194651731.

\section{Data availability statement}

The data that support the findings of this study are available upon reasonable request from the authors.

\section{ORCID iDs}

I J Bermúdez Macias (D) https://orcid.org/0000-0002-28424230

S Düsterer (D) https://orcid.org/0000-0003-4379-1327

R Ivanov (D) https://orcid.org/0000-0002-6716-5104

U Frühling (D) https://orcid.org/0000-0002-8423-786X

N M Kabachnik (D) https://orcid.org/0000-0003-4458-7643

\section{References}

[1] Kuchiev M Y and Sheinerman S A 1989 Usp. Fiz. Nauk 158 353-87

[2] Mehlhorn W 1998 J. Electron Spectrosc. Relat. Phenom. 93 $1-15$

[3] Schmidt V 1997 Electron Spectrometry of Atoms Using Synchrotron Radiation (Cambridge: Cambridge University Press)

[4] Eberhardt W, Bernstorff S, Jochims H W, Whitfield S B and Crasemann B 1988 Phys. Rev. A 38 3808-11

[5] Aksela H, Kivilompolo M, Nõmmiste E and Aksela S 1997 Phys. Rev. Lett. 79 4970-3

[6] Armen G B, Southworth S H, Levin J C, Arp U, LeBrun T and MacDonald M A 1997 Phys. Rev. A 56 R1079-82

[7] Lablanquie P, Sheinerman S, Penent F, Hall R I, Ahmad M, Hikosaka Y and Ito K 2001 Phys. Rev. Lett. 87 053001

[8] Hentges R, Müller N, Viefhaus J, Heinzmann U and Becker U 2004 J. Phys. B: At. Mol. Opt. Phys. 37 L267-73

[9] Hergenhahn U, Fanis A D, Prümper G, Kazansky A K, Kabachnik N M and Ueda K 2005 J. Phys. B: At. Mol. Opt. Phys. 38 2843-57

[10] Niehaus A 1977 J. Phys. B: At. Mol. Phys. 10 1845-57

[11] Morgenstern R, Niehaus A and Thielmann U 1977 J. Phys. B: At. Mol. Phys. 10 1039-58

[12] Ogurtsov G N 1983 J. Phys. B: At. Mol. Phys. 16 L745-8

[13] Russek A and Mehlhorn W 1986 J. Phys. B: At. Mol. Phys. 19 911-27

[14] Armen G B, Tulkki J, Aberg T and Crasemann B 1987 Phys. Rev. A 36 5606-14

[15] Tulkki J, Åberg T, Whitfield S B and Crasemann B 1990 Phys. Rev. A 41 181-5

[16] Kuchiev M Y and Sheinerman S A 1985 J. Phys. B: At. Mol. Phys. 18 L551-6

[17] Sheinerman S A 2003 J. Phys. B: At. Mol. Opt. Phys. 36 4435-46

[18] Kazansky A K and Kabachnik N M 2005 Phys. Rev. A 72 052714

[19] Drescher M et al 2002 Nature 419 803-7

[20] Drescher M, Hentschel M, Kienberger R, Uiberacker M, Westerwalbesloh T, Kleineberg U, Heinzmann U and Krausz F 2004 J. Electron Spectrosc. Relat. Phenom. 137-140 259-64 iCESS-9 Proceedings of the 9th International Conference on Electronic Spectroscopy and Structure

[21] Uiberacker M et al 2007 Nature 446 627-32

[22] Uphues T, Schultze M, Kling M F, Uiberacker M, Hendel S, Heinzmann U, Kabachnik N M and Drescher M 2008 New J. Phys. 10025009

[23] Krikunova M et al 2009 New J. Phys. 11123019

[24] Verhoef A J, Mitrofanov A V, Nguyen X T, Krikunova M, Fritzsche S, Kabachnik N M, Drescher M and Baltuška A 2011 Laser Phys. 21 1270-4

[25] Zherebtsov S et al 2011 J. Phys. B: At. Mol. Opt. Phys. 44 105601

[26] Schütte B et al 2012 Phys. Rev. Lett. 108253003

[27] Ackermann W et al 2007 Nat. Photon. 1336-42

[28] Bauch S and Bonitz M 2012 Phys. Rev. A 85053416

[29] Trinter F et al 2013 Phys. Rev. Lett. 111093401

[30] Ivanov R, Liu J, Brenner G, Brachmanski M and Düsterer S 2018 J. Synchrotron Radiat. 25 26-31

[31] Ivanov R et al 2020 J. Phys. B: At. Mol. Opt. Phys. 53 184004

[32] Kazansky A K, Sazhina I P and Kabachnik N M 2009 J. Phys. B: At. Mol. Opt. Phys. 42245601

[33] Frühling U 2011 J. Phys. B: At. Mol. Opt. Phys. 44 243001 
[34] Stolterfoht N, Gabler H and Leithäuser U 1973 Phys. Lett. A 45 $351-2$

[35] Goldsztejn G et al 2017 Phys. Rev. A 96012513

[36] Carroll T X, Bozek J D, Kukk E, Myrseth V, Sæthre L J and Thomas T D 2001 J. Electron Spectrosc. Relat. Phenom. 120 $67-76$
[37] Werme L O, Bergmark T and Siegbahn K 1972 Phys. Scr. 6 $141-50$

[38] Penent F, Palaudoux J, Lablanquie P, Andric L, Feifel R and Eland J H D 2005 Phys. Rev. Lett. 95083002

[39] Decking W et al 2020 Nat. Photon. 14 391-7

[40] Emma P et al 2010 Nat. Photon. 4 641-7 